Original Research Paper

\title{
Experimental Study: The Effects of Mobile Phone Icons Characteristics on Users' Age Groups
}

\author{
${ }^{1}$ Syed Ghayas, ${ }^{2}$ Salim Amor Al-Hajri and ${ }^{3}$ Suziah Sulaiman \\ ${ }^{1,3}$ Department of Computer and Information Sciences, Universiti Teknologi PETRONAS, Malaysia \\ ${ }^{2}$ Department of Management Information Systems, A'Sharqiyah University, Ibra, Oman
}

\author{
Article history \\ Received: 06-06-2017 \\ Revised: 05-07-2017 \\ Accepted: 06-08-2018 \\ Corresponding Author: \\ Syed Ghayas \\ Department of Computer and \\ Information Sciences, \\ Universiti Teknologi \\ PETRONAS, Malaysia \\ Email: syedghayas@ciit.net.pk
}

\begin{abstract}
Mobile phone applications interfaces offer great support to various age group users. There is a lot of Mobile Human Computer Interaction (here after $\mathrm{HCI}$ ) research work going on, which is found in User Experience - UX issues. Until now little research work is done on Mobile phone icons and its effect on learn ability for various age group users. The aim of this study is to investigate the effects of mobile phone icons characteristics on users' age groups. In current research work we carry out an exploratory study followed by an in-depth experimental based study in order to find out which icons characteristics effect icon usability for various age group users. From experimental results, it is revealed that elderly have more issues while interpreting mobile phone icons, but specifically icon characteristics-semantic distance, familiarity and complexity- are having potential in order to improve icon user experience. We further discuss how design guidelines can support application designers to design application icons that can be adopted by various age group users and be beneficial for them.
\end{abstract}

Keywords: Mobile Phone Icons, User Experience, Age Groups

\section{Introduction}

Icons are essential component of user interfaces, as they turn into a gateway to the vast number of functionalities for such mobile devices. As there is a growth in new technologies and platforms, there is a growth in number of users who use those technologies. Many users lack skills in mobile phone technologies as they are unable to understand and operate those applications (Wilkinson et al., 2013).

The end users are the main target of the market producing new platforms and technologies. It is observed that little research work has been carried out on the influence of graphical icons on various age group users when using modern mobile phone technologies (Ghayas et al., 2013a). As age grows, there is effect on cognition and perception capabilities which results effecting elderly users' ability in interpreting icons (Marcus, 2013).

Initial user experience with icons is an important factor for learn ability in order to use mobile technology because of the diverse features, functionalities and user interfaces available on mobile platforms (Gatsou et al., 2012). Mobile devices functionalities differ from what is offered in computer desktop or laptop domain. Some of the examples are text entry methods and Internet options. Majority of those functionalities can only be retrieved using special designed icons and buttons. As mobile phones have specific operating systems (such as Symbian, Windows Mobile, Palm OS, Blackberry OS and I phone IOS) and each operating system provides its own set of designed icons. Initial user experience with icons is critical for mobile phones, reason is that they appear to develop rapidly, thus the users are inclined to change mobile phones more constantly as compared to computers.

Many icons design guidelines have been published. Some established examples are: "Icons and image design for IOS" (Apple, 2014), "Andriod Iconography" (Android, 2014), "Icons should be suggestive of the functionality which they are associated" (Benson et al., 2010) and "Icons and familiarity level" (Heim and Heim 2008). These guidelines are general in nature and have been applied to all age group users. However, until now it is not 
known whether these existing universal design guidelines as claimed by others (Kobayashi et al., 2011; Gatsou et al., 2012; Mäkelä, 2015) are applicable to age related differences. The majority of design guidelines are only applicable to younger population (Haverila, 2013, Mäkelä, 2015), thus, ignoring the elderly. The motivation of our research work is to explore whether these existing design guidelines can be generalized for age related difference and hence, can be adopted for the elderly.

The objective of this research paper is to prove experimentally the influence of icons characteristics on various age group in term of icon user experience. From the qualitative part of our previous study we identify the icons characteristics which either obstruct or help user experience (Ghayas et al., 2014). The study was conducted in order to understand the various age related difference in icons usability and identification of icons characteristics which is affecting user experiences for elderly. The results from our exploratory study are the important icon characteristics which are complexity, familiarity and semantic distance. In order to investigate in detail, the effect of each of icon characteristics on the icons user experiences, we extend the study and further explore with the help of experiment in order to see the effect of characteristics on various age group users i.e., with younger and older adults. This is the central essence of this paper. Since there is little literature available on icons interpretability issues that elderly experience (Hawthorn, 2005; Siek et al., 2005). In this research work, we will focus on the initial learning and learning in long term (extended learning) while learning mobile phone icons.

\section{Literature Review}

The aim of this study is to investigate the effects of mobile phone icons characteristics on users' age groups. The review of past studies will enable us to explore the design icons and its characteristics which are associated with overall user experience and also the user experience of the application in which the icon is used. For example, Fig. 1 below shows some examples of these icons from two different mobile platforms.

Past research work (Heim and Heim, 2008), reports that there are a few icons characteristics identified which affect its usability. Those characteristics are visual detail, color and size. It also includes the icons choices and meanings. Other study (Horton, 1994), focuses on user characteristics (e.g., user intelligence, experience and cultural background) and in the domain in which the icon is found (e.g., User interface, mobile device, functions) as factors that influence icon usability. Similarly, there are a few other studies which explore the overall effects on icon usability of some of these characteristics, for example, animation (Baecker et al., 1991) and spacing and size (Lindberg and Näsänen, 2003). While most of these works are related to the physical characteristics, less is done on other aspects of icons characteristics and its effect on users' perception.

According to a research study (McDougall et al., 2000), pertaining to the effects of icon characteristics (concreteness and complexity) on different tasks involved, search and linking icons with labels has been investigated. It reports that test participants of the study found is very difficult in order to interpret abstract icons as compared to concrete icons. The researcher recommends that the use of concrete icons increase the learn ability of interfaces.

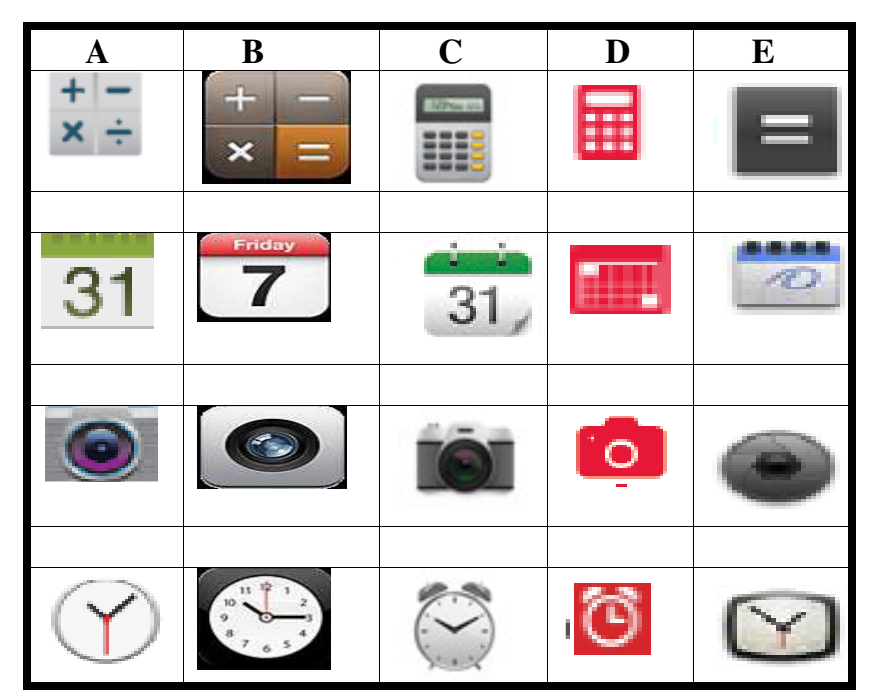

Fig. 1: Sample icon list 
As reported in a research wok (Wiedenbeck 1999) on how labels effect on icon usability, test participants did better with labels provided with text than labels without text. This study's results reveal that test participants perform well with labels than icons without labels. This indicates that it is very easy to use labeled icons.

Even though not many have focused on icon characteristics on mobile phones, there are quite a number who have studied icons in different contexts such as icons for the road signs and medical signs. Some studies (Rogers 1989; McDougall et al., 2000) reveals that concrete icons are preferred than those are abstract to the users. The concreteness of an icon should be the same with that in the real world. If familiarity with icons increased, the benefits of icon concreteness minimize (McDougall et al., 2000; Isherwood et al., 2007). The familiarity of an icon depends on how much a user is more informed about a particular icon. Also identifying visually complex icons comes along with long recognition and encoding times (Arend et al., 1987). In a research work done by Forsythe et al. (2008) on icons usability who states that icon familiarity and complexity are correlated and it is very easy for users to interpret familiar icons.

In this paper, our research work is based on several Leung, 2009; Gatsou et al., 2012), which basically explore the effect of concreteness, labels and semantic distance on various age group users. These researchers were specifically looking into initial icon usability. According to other research work (Rogers and Oborne, 1987; Green and Barnard 1990; Stotts, 1998), icon identification is the key element of a task in which the concreteness will affect the user perception when identifying icons. The reason is that concrete icons depict objects, allowing people to use their knowledge of the everyday world in order to interpret them but this is less easily done with abstract icons. We further extend the above reported work by not only looking into initial icon usability, but using more depth based strategy and interested to find out what the effects will be in long term use of those icons. The interesting situation is that, now we have third generation of mobile phone icons. Those icons are basically the operating system provided icons. In summary, we need to explore the overall mobile phone icons users experience including the operating system provided icons.

\section{Qualitative Exploratory Study Methodology}

In order to find out the Effects of Mobile Phone Icons Characteristics on Users' Age groups perceptions, we carried out a qualitative exploratory study reported in our previous experiment (Ghayas et al., 2013b). This study involves 25 users representing the older and younger groups. All users have basic knowledge of computers and mobile phones. Icons from two mobile phones brands are used in the study which are: (i) Motorola droid Bionic and (ii) Samsung Galaxy. The first stage of the study involves the test participants interpreting the icons provided on both phones and how they associate them to the real world objects. Participants were then invited to examine each icon then identify the object they saw on that particular icon and say out loud which mobile phone functionality should be associated with it. In the second stage, participants were asked to carry out a series of tasks on each provided phone. For example: Locating icons for a specific program (e.g., Wi-Fi program) or functionality (e.g., settings). From the study, important findings of the icon characteristics and its effects on various age group users' were identified. These icon characteristics are complexity, familiarity and semantic distance. The details of this study have been reported in the research work on icons characteristics (Ghayas et al., 2013b).

\section{Experimental Study Methodology}

Based on the qualitative exploratory study described above, we setup a detailed experiment in order to do an in-depth investigation to what level familiarity, complexity and semantic distance affect initial icon user experience in various age group users.

The hypotheses of the experiment were identified as follows:

- Hypothesis 1: Elderly users encounter more difficulties in overall icon interpretation of mobile phone than younger adults

- Hypothesis 2: Elderly users encounter more difficulties in terms of interpreting complex icons of mobile phone than younger adults

- Hypothesis 3: Elderly users encounter more difficulties in distinguishing semantically close mobile phone icons than younger adults

- Hypothesis 4: Younger adults are more familiar with the mobile phone icons than the elderly

\section{Experimental Design}

In our experimental design we used 4-factor mixed design method: two age groups (20-40 and 50+), familiarity, complexity and semantic distance which are all with-in subject factors.

\section{Test Participants}

Two groups of equal numbers were formed, consisting of 60 test participants in total. The first group consists of those participants ranging from 20 to 40 years old (younger adults) while the second from $50+$ years of age (older adults). Test participants were invited through departmental advertisement boards. It was a pre-requisite for all the participants that they should have some experience with usage of mobile phones, correct eyesight, no color blindness and fluency in English. 
Before conducting the study a few tests were conducted on to the test users, such as vision test and verbal fluency. The test mechanism used was Snellen eye test and the FAS test (Benton and Hamsher, 1983), respectively. The results confirmed that the test users had normal vision and meeting verbal fluency levels as required for conducting the study.

\section{Measures}

Data were collected to test our hypothesis. Each icon tested was given a score. This is in association with the responses from questions and icons provided in survey. The score is obtained for the following dependent variables:

1. The degree on how accurate the icon is while identification procedure of icons is taking place. (Values to be used: 0 or 1 )

2. The degree of accuracy in interpreting the icons. (Values to be used: 0 or 1 )

3. The confidence level of the participants in interpreting the icons meaning (values to be used: scale $1-5 ; 1=$ no sure; $5=$ very much assure)

4. The degree of familiarity with the icons (values from 1-5)

In our experimental study, we focused on the initial usability required for mobile phone interfaces and then the long term use of them. Familiarity scores of the test users with icons in our study were measured in order to assess and control the effect of the icons on the initial user experience measures.

\section{Materials}

We selected icons from different mobile phones that are popular in year 2013. Mobile phones brands selected were Samsung Galaxy S3, Samsung Nexus, Apple i-Phone, Blackberry bold and Nokia Lumia. All icons taken from above phones are presented in a list shown in Fig. 1 (sample icons list). We enlarged most of the icons to minimize effects of icons sizes due to differences in vision capabilities among the participants. All icons were displayed on the computer screen when presenting them to the test participants. Four students from Human computer interaction cluster rated the complexity, familiarity and semantic distance of all the provided icons in experiment.

\section{Procedures and Study Samples}

The study was mainly carried out in the HCItesting lab located at Computer and Information Sciences Department, University Technology PETRONAS while a few of the sessions were conducted at the participants' places. We also visited community centers in order to carry out test with elderly. A consent form was given before conducting the study. All participants were requested to carefully read the consent form before signing it. The first ten minutes was given to familiarize test users with the functionalities of existing modern mobile phones (e.g. camera programme and using the Internet).

In the main study session, each participant was shown the icons list and was given the questions for each single icon that was presented. The participant has to rate each icon with regards to the question prepared. The following are the sample questions asked during the experiment from the test participants pertaining to the icons:

1. Identification of the icon objects - (example: What can you see in the icon?)

2. Interpreting the icon's functionality - (example: How can we use the icon?)

3. Level of familiarity with the icon - (example: Have you experience this icon before?)

4. Confidence level while interpreting icons - (example: Are you sure on the functionality of the icon?)

\section{Statistics Scores}

The scores of test participants understanding and identification of icons were recorded. Also interpretation of the icons meanings was scored during the test session. Three HCI experts rate the test scores for the experiment. We calculated agreement percentage of how icons identified for both groups. Scores were $80 \%$ and $88 \%$ respectively.

In order to assess the measure of icon identification and interpretation, the raters focused more on the description of thoughts. On the other hand, raters avoid the use of exact words or phrases. Example is, if a user has been asked to open a camera programme, responses with either to open camera programme or icon for a camera application would be scored as correct. During test, we are not supposed to let test users know about their response either correct or incorrect.

\section{Results and Analysis}

The aim of this study is, to investigate the effects of mobile phone icons characteristics on users' age groups. To achieve the above aim, we used Cramer's $\mathrm{V}$ test in order to measure the strength, level of association and effect sizes between our experimental variables. The sample interpretation of association or effect sizes is described in Table 1.

Based on the results from SPSS-16, the analysis provides no association (or no differences) between Complexity and Age group (younger Vs older) regarding icons $A$ and $E$. In addition, the measured effect sizes presented a medium level of association ( $\mathrm{V}=0.421$ for $\mathrm{A}$ ). But there is real association (or difference) exists between Complexity and Age group regrding icons $\mathrm{B}, \mathrm{C}, \mathrm{D}($ as $p<0.05)$ and also with high effect sizes $(0.681,0.596,0.655)$. 
Icons for calendar there is medium level of association ( $\mathrm{V}=0.435$ for $\mathrm{D}$ and $\mathrm{V}=0.461$ for $\mathrm{E})$ and real association between complexity and age group regarding icons $\mathrm{A}, \mathrm{B}, \mathrm{C}$ (as $\mathrm{p}<0.05)$ and high effect sizes of $(0.694,0.694,0.694)$ respectively.

To further our analysis, we used ANOVA to test our hypothesis. The gold standard statistical argument (Cohen, 1988) of inferential statistics is considered. We run a 2 (age group) *2(semantic distance) *2(complexity) *2(familiarity) ANOVA in order to find out icon object identification accuracy. In order to measure both how icon interpreted and users' confidence while interpretation, we design and run a 2(age groups) $* 2$ (semantic distance) $* 2$ (complexity) $* 2$ (familiarity) ANOVA test.

When a statistical significant result was found, we followed up with post hoc pair wise comparisons, using Bonferroni correction to protect against type I error. Using this correction method resulted in degrees of freedom that are not whole numbers. In our analysis we also report the eta-squared $\left(\mathrm{h}^{2}\right)$ statistics. In statistics etasquared is a extent of effect size, which provide more detailed information as compared to statistical significance in applied human-computer interaction research (Landauer, 1997).

In order to interpret this value, the author (Cohen, 1988) mentioned that $0.01,0.06$ and 0.14 are considered small, medium and large effect sizes, respectively.

\section{Measuring Level of Confidence and Familiarity}

When mean and standard deviation is compared, we can see that confidence was relatively low while interpreting icons. While recording scores for user's confidence, we only record for correct meaning. There are few cases where none of users correctly interpret any icon. Here we did not record any confidence scores for them. It also reflects the statistical findings.

We explore the users' level of understanding (perception) with the given icons in order to see the effect of users' experience. According to ANOVA of familiarity scores it is revealed that younger adults are more familiar (7 out of 10 ) than older adults (5 out of 10$)$. We also explore the overall effect of familiarity on our dependent variables. From results we see that familiarity is correlated with our dependent variables (Details in Table 2).

If we have to look on the measures we can see that familiarity have some effect on our statistical scores. In further analysis we also run the ANOVA controlling its effects by dealing it as a covariate. The results reveal that with controlled familiarity the significance effect on independent variables remains.

\section{Overall User Experience (UX)}

According to Hypothesis no 1, as compared to younger adults our older adults were not confident while icon identification and interpretation. Both scores vary significantly from each other as shown in Table 3.
Scores of Interpretation confidence also differ significantly between the two age groups. Both groups of participants differ in terms of confident levels while interpreting icons. The results are summarized in Table 3.

\section{Overall Effect of Complexity, Familiarity and Semantic Distance}

Icons identification - From the experimental results we found that age related effects on icon familiarity, complexity and semantic distance. We discussed further how users identified and interpreted icons Fig. 2.

The elderly performed worst when identifying and interpreting complex icons which supports hypothesis 2 . All statistical scores for complexity, familiarity and semantic distance are presented in Table 3.

\section{Icons Interpretation}

There is a significant difference in interpretation of icons between younger and elderly users. The younger users are better in interpreting icons than elderly users see Figure 3.

According to hypothesis 4 , there are significant age- related effects in both groups in term of familiarity while icon interpretation. As the younger users were more familiar with icons than elderly, so it is very easy for them to interpret.

Both age group users find it easy to interpret semantic close icons $(p<0.001)$. Age-related effect was found when interpreting between complex icons.

\section{Icons Interpretation and Overall Confidence}

There is a significant effect of two age groups on semantic distance, familiarity and complexity. The younger were more familiar than elderly users; hence they were more confident in order to interpret icons than the elderly. Both groups have same confidence levels when interpreting semantically close icons.

\section{Summary}

Our experimental study reveals the following results related to our hypothesis:

- Hypothesis 1 Supported: Elderly users encounter more difficulties in overall icon interpretation of mobile phone than younger adults.

- Hypothesis 2 Supported: Elderly users encounter more difficulties in terms of interpreting complex icons of mobile phone than younger adults.

- Hypothesis 3 Supported: Elderly users encounter more difficulties in distinguishing semantically close mobile phone icons than younger adults.

- Hypothesis 4 (familiarity) Supported: Younger adults are more familiar with the mobile phone icons than the elderly. 


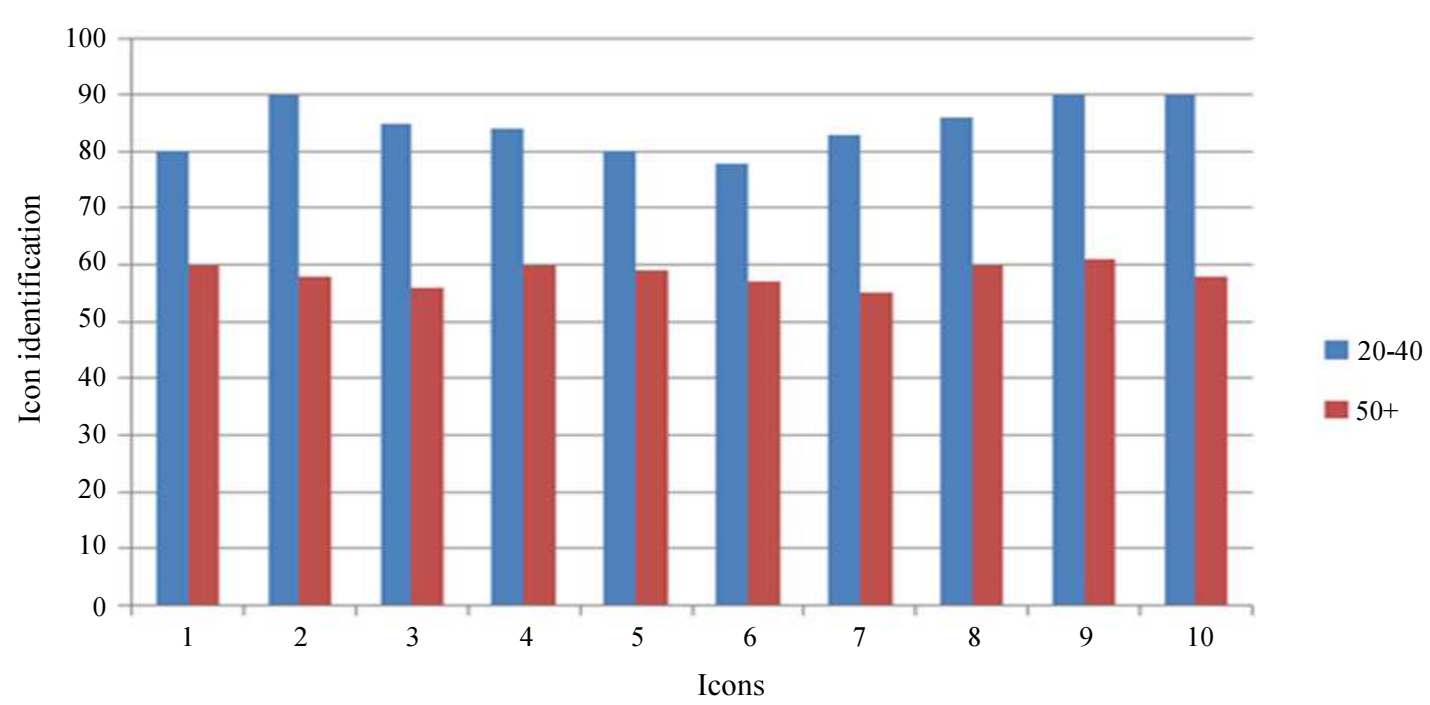

Fig. 2: Icon identification by age groups

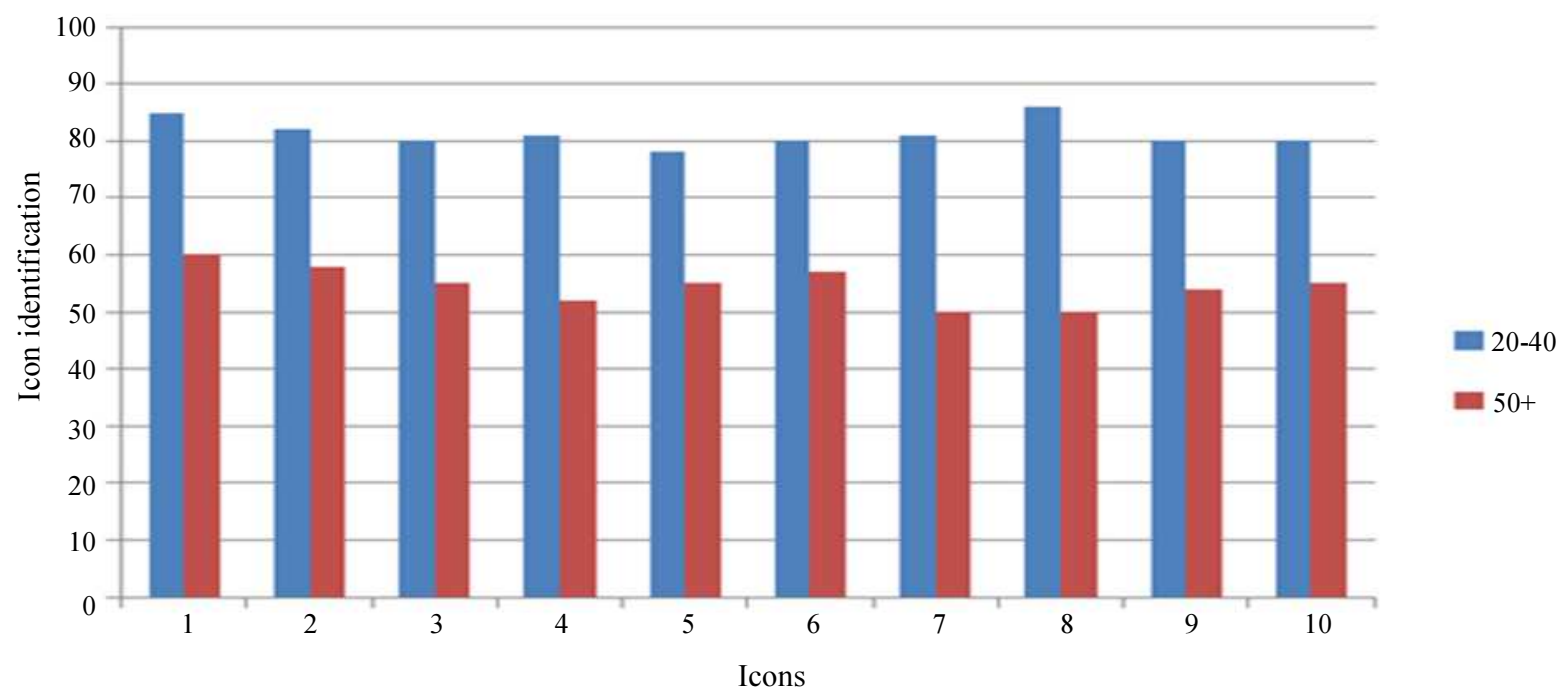

Fig. 3: Icon interpretation by age groups

Table 1: Measure of effect size and Association

\begin{tabular}{llllllll}
\hline & & \multicolumn{2}{l}{ Complexity } & & Familiarity & & \multicolumn{2}{l}{ Semantic distance } \\
& Icon & Cramer's V & P-value & Cramer's V & P-value & Cramer's V & P-value \\
\hline \multirow{3}{*}{ Calculator } & A & 0.421 & 0.069 & No association & No association & 0.561 & 0.002 \\
& B & 0.681 & 0 & 0.733 & 0 & 0.682 & 0 \\
& C & 0.596 & 0.007 & 0.451 & 0.017 & 0.503 & 0.006 \\
& D & 0.655 & 0.001 & 0.599 & 0.002 & 0.503 & 0.017 \\
& E & No association & No association & 0.333 & 0.035 & No association & No association \\
& A & 0.694 & 0 & 0.5 & 0.007 & 0.951 & 0 \\
& B & 0.694 & 0 & 0.507 & 0.016 & 0.777 & 0 \\
& C & 0.649 & 0.002 & 0.531 & 0.01 & 0.636 & 0.001 \\
& D & 0.435 & 0.023 & 0.478 & 0.028 & No association & No association \\
& E & 0.461 & 0.014 & 0.5 & 0.4 & No association & No association \\
\hline
\end{tabular}


Table 2: Pearson correlation Measure (Familiarity and icons user experience measure)

\begin{tabular}{lllll}
\hline & $\begin{array}{l}r \text { person's } \\
\text { correlation coefficient }\end{array}$ & $\begin{array}{lll}r^{2} \text { coefficient of } \\
\text { determination }\end{array}$ & P-value & $\mathrm{N}$ \\
\hline Icon identification score & 0.22 & 0.04 & $<0.001$ & 30 \\
Icon interpretation score & 0.35 & 0.12 & $<0.001$ & 30 \\
Overall confidence score & 0.30 & 0.09 & $<0.001$ & 30 \\
\hline
\end{tabular}

Table 3: Mean, Standard deviation icon user experience scores, for complexity, familial semantic distance $(\mathrm{N}=60)$

\begin{tabular}{|c|c|c|c|}
\hline & Complexity & Familiarity & Semantic distance \\
\hline \multicolumn{4}{|c|}{ Icon identification } \\
\hline $20-40$ & $58 \%(60 \%)$ & $80 \%(8 \%)$ & $82 \%(10 \%)$ \\
\hline $50+$ & $55 \%(10 \%)$ & $50 \%(15 \%)$ & $60 \%(12 \%)$ \\
\hline \multicolumn{4}{|c|}{ Interpretation accuracy } \\
\hline $20-40$ & $77 \%(15 \%)$ & $80 \%(11 \%)$ & $78 \%(10 \%)$ \\
\hline $5+$ & $55 \%(17 \%)$ & $50 \%(13 \%)$ & $48 \%(12 \%)$ \\
\hline \multicolumn{4}{|c|}{ Overall confidence } \\
\hline $20-40$ & $3.38(0.37)$ & $3.40(0.44)$ & $3.35(0.45)$ \\
\hline $50+$ & $3.20(0.47)$ & $3.18(0.58)$ & $3.18(0.52)$ \\
\hline
\end{tabular}

\section{Discussion}

Findings show support for the above hypothesis as follows:

\section{Overall Users Experience with Existing Icons}

From experimental results it is revealed that elderly have difficulty while interpreting mobile phone icons. The findings are matching with the authors Leung et al. (2009; Cherng et al., 2016). The modern operating system provided icons are both difficult for elderly and younger to interpret correctly. Interpreting icons greatly affect the overall learn ability of interfaces. We can conclude and suggest that there is a need to redesign icons to make it more learnable for both age group users.

From the experiment we found that complex icons and icons which are not familiar for both age groups are difficult to interpret. In the next section we will discuss the findings from the experiment and then derive design guidelines for icons which will improve the overall learn ability.

\section{Effect of Complexity on Icons Interpretability}

From the experimental results, it is revealed that both age group users have difficulties while identifying and interpreting complex designed icons (Schröder and Ziefle, 2008; Leung et al., 2009; Cherng et al., 2016).

\section{Effect of Semantic Distance on Icons Interpretation}

From our experimental results it is revealed that it is very difficult for both groups to interpret icons which have semantically not close. From results we can see that semantic distance has larger effect on improving the learn ability of mobile phone applications interfaces (Leung, 2009). The reason why it is hard for the users to interpret semantic far icons is the reason reported in literature (Ziefle and Bay, 2005; Leung et al., 2010; Cherng et al., 2016). Many users greatly rely on understanding of how system works and operates. Icons with semantic far meaning need exact mental model of the system that the user can apply while interpreting icons. Examples are user need to know if a mobile phone can perform call in order to interpret call icon. Users from both groups have higher chances of difficulty when new functions were introduced to them.

From our experimental results icons with semantic far meaning are difficult for both groups. But that ratio is higher amongst elderly. One solution to overcome semantic distance characteristics is design icons having objects with close semantic meaning. Following are alternates to reduce semantic distance.

\section{Icon Designed with Close Real World Objects}

From our experimental results we can see that more familiar the users are with the icons, the better users' experience they have with it. We review the existing guidelines for mobile phone icons which suggest and emphasis using familiar metaphors and images to represent icons. If it is not possible to design icons with familiar metaphors, then the enough training is provided to the users about metaphors and interfaces. Most of our users are very interested to learn the modern icons and interfaces of mobile phones.

There is ongoing discussion that there will be no issue in familiarity with mobile phone icons in the future when the upcoming generations which will be having much more experience with mobile phone icons and interfaces. According to a research work (Leung et al. 2012), it was reported that there are a lot of younger users which still did not interact with modern mobile application interfaces. Many of our elderly users report that they do not need the continual learning of modern mobile phone applications interfaces. As there is a rapid growth in mobile phone icons development. So there are more chances that users will be having difficulty to learn about it and be familiar with it. 


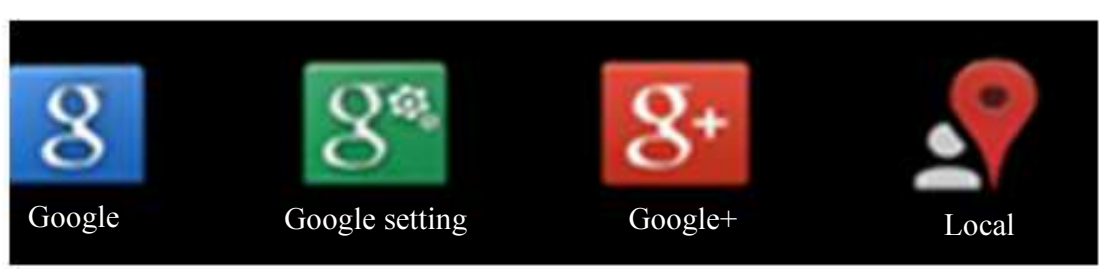

Fig. 4: Operating system specific icons

\section{Designing Operating System Provided Icons}

As most of mobile operating system is providing its own set of icons, it is revealed in our experiment that it is very difficult for both the group users to interpret operating system provided icons. Example Fig. 4, we suggest that operating system provided icons should be grouped together, which effect user's perception and improve their level of familiarity with those kinds of icons.

As these icons are third generation icons, so as most of our younger and elderly will be unfamiliar with these icons. Even the users using specific platform were unfamiliar with it. Most of users replied that they are unaware of those icons. That can be improved by grouping all those icons together, as those icons work in similar domain.

\section{Icons Selection by Users}

According to the experimental results, it is revealed that icons designed and understand by one user could be different as perceived by other person. We propose guideline that mobile icons should not just provide different functions. The icon can be adaptive and hence fit to user's context, e.g., is Internet icon. There are various images/shapes which can represent an Internet icon. It is more suitable if users to be empowered that they can chose to select icons which can be fit to their context. We expect that if users are able to choose their icons, will improve overall user experience.

\section{Conclusion}

In the current experimental study, our objective was to find out the effects of icon characteristics on various age group users, in order to have in-depth idea what design strategies to use to better design icons. We also come up with icon design guidelines which can fit according to user preference and results in better understand mobile interface and also improve its learn ability. In first part of our work we conducted a qualitative exploratory study and found that younger and older adults face issues while working with mobile phone icons. There are few icon characteristics identified which effect overall icon user experience.

In second step we carry out a detailed experimental study in order to understand the effects of icon characteristics which are complexity, familiarity and semantic distance. From our experimental study we found out that those characteristics affect icon user experience. Results revealed that it is easy for younger adults to interpret most of icons as compared to elderly.

Semantic close icons are easy for elderly to interpret, but they have issues in interpreting complex and less familiar icons. Operating system provided icons are very difficult for both the groups to interpret. It is revealed that younger adults are much familiar with icons than elderly.

From our experimental study it shows that existing icons should be redesigned for both age group users in order to improve learn ability. We also derived few icon design guidelines which will help application designer to understand age specific needs and design icons which will be more learnable.

We also suggest the design of semantic close icons and icon which are more familiar for users. Operating system provided icons should be group together. Our experimental results are consistent with many industrial guidelines and standards. Our results can be integrated with current practices in order to improve the learn ability of both age group users using mobile phone applications.

Current and future mobile phone offers a lot of opportunities for younger and older adults to learn interfaces and be active user. The more will mobile phone icons are easier to learn; it will affect the overall user experience of both age group users.

\section{Implication, Limitations and Future Research}

In this study we presented the icons on the real mobile phones. This might have some effect on the results if these icons are presented on paper or on a computer screen. Future work in the domain will need to see the extent to which results change, if the medium which icons should be presented to users are updated.

\section{Author's Contributions}

All authors equally contributed in this work.

\section{Ethics}

This article is original and contains unpublished material. The corresponding author confirms that all of the other authors have read and approved the manuscript and there are no ethical issues involved. 


\section{References}

Android, 2014. Android icon design guidelines.

Apple, 2014. Icon design guidelines.

Arend, U., K.P. Muthig and J. Wandmacher, 1987. Evidence for global feature superiority in menu selection by icons. Behav. Inform. Technol., 6: 411-426. DOI: 10.1080/01449298708901853

Baecker, R., I. Small and R. Mander, 1991. Bringing icons to life. Proceedings of the SIGCHI Conference on Human Factors in Computing Systems, Apr. 27-27, ACM New Orleans, Louisiana, pp: 1-6.

DOI: $10.1145 / 108844.108845$

Benson, C., B. Clark and S. Nickell, 2010. Designing effective icons. GNOME Human Interface Guidelines.

Benton, A.L. and K.S. Hamsher, 1983. Multilingual aphasia examination: Manual of instructions. University of Iowa.

Cherng, F.Y., W.C. Lin, J.T. King and Y.C. Lee, 2016. An eeg-based approach for evaluating graphic icons from the perspective of semantic distance. Proceedings of the 2016 CHI Conference on Human Factors in Computing Systems, May 07-12, ACM, San Jose, California, pp: 4378-4389. DOI: $10.1145 / 2858036.2858133$

Cohen, J., 1988. Statistical Power Analysis for the Behavioral Sciences. 2nd Edn. Hillsdale, NJ: Erlbaum.

Forsythe, A., G. Mulhern and M. Sawey, 2008. Confounds in pictorial sets: The role of complexity and familiarity in basic-level picture processing. Behav. Res. Meth., 40: 116-129.

DOI: 10.3758/BRM.40.1.116

Gatsou, C., A. Politis and D. Zevgolis, 2012. The Importance of Mobile Interface Icons on User Interaction. Int. J. Comput. Sci. Applic., 9: 92-107.

Ghayas, S., S. Sulaiman, J. Jaafar, S. Mahamad and M. Khan, 2014. Mobile phone Icons recognition and cultural aspects. Proceeding of the International Conference on Computer and Information Sciences, Jun. 3-5, IEEE Xplore Press, Kuala Lumpur, Malaysia, pp: 1-5.

DOI: $10.1109 /$ ICCOINS.2014.6868833

Ghayas, S., S. Sulaiman, M. Khan and J. Jaafar, 2013a. The effects of icon characteristics on users' perception. Proceedings of the 3rd International Visual Informatics Conference on Advances in Visual Informatics, Nov. 13-15, Springer, Selangor, Malaysia, pp: 652-663.

DOI: 10.1007/978-3-319-02958-0 59

Ghayas, S., S. Sulaiman, M. Khan and J. Jaafar, 2013 b. Qualitative study to identify icons characteristics on mobile phones applications interfaces. Proceeding of the IEEE Symposium on Wireless Technology and Applications, Sept. 22-25, IEEE Xplore Press, Kuching, Malaysia, pp: 310-315.

DOI: 10.1109/ISWTA.2013.6688794
Green, A.J.K. and P.J. Barnard, 1990. Iconic interfacing: The role of icon distinctiveness and fixed or variable screen locations. Proceeding of the IFIP TC13 3rd International Conference on Human-Computer Interaction, Aug. 27-31, Cambridge, UK.

Haverila, M., 2013. Cell phone usage and broad feature preferences: A study among Finnish undergraduate students. Telemat. Inf., 30: 177-188.

DOI: $10.1016 /$ j.tele.2012.05.002

Hawthorn, D., 2005. Training wheels for older users. Proceedings of the 17th Australia Conference on Computer-Human Interaction: Citizens Online: Considerations for Today and the Future. Nov. 21-25, Computer-Human Interaction Special Interest Group (CHISIG) of Australia, Canberra, Australia, pp: 1-10.

Heim, S. and S.G. Heim, 2008. The Resonant Interface: HCI Foundations for Interaction Design, Pearson/Addison Wesley.

Horton, W.K., 1994. The ICON Book: Visual symbols for computer systems and documentation. John Wiley and AMP.

Isherwood, S.J., S.J. McDougall and M.B. Curry, 2007. Icon identification in context: the changing role of icon characteristics with user experience. Hum Factors, 49: 465-476. DOI: 10.1518/001872007X200102

Kobayashi, M., A. Hiyama, T. Miura, C. Asakawa and M. Hirose, 2011. Elderly user evaluation of mobile touchscreen interactions. Proceedings of the 13th IFIP TC 13 International Conference on Human-Computer Interaction, Sep. 05-09, Springer-Verlag Berlin, Lisbon, Portugal, pp: 83-99.

Landauer, T.K., 1997. Behavioral Research Methods in Human-Computer Interaction. In: Handbook of Human-Computer Interaction, Helander, M.G., T.K. Landauer and P.V. Prabhu (Eds.), Elsevier, New York ISBN-10: 0080532888, pp: 203-227.

Leung, R., 2009. Improving the learnability of mobile device applications for older adults. CHI '09 Extended Abstracts on Human Factors in Computing Systems, Apr. 04-09, ACM Boston, MA, USA, pp: 3125-3128. DOI: $10.1145 / 1520340.1520443$

Leung, R., L. Findlater, J. McGrenere, P. Graf and J. Yang, 2010. Multi-layered interfaces to improve older adults\&rsquo; initial learnability of mobile applications. ACM Trans. Access. Comput., 3: 1-30.

Leung, R., J. McGrenere and P. Graf, 2009. Age-related differences in the initial usability of mobile device icons. Behaviour Inform. Technol., 30: 629-642. DOI: $10.1080 / 01449290903171308$

Leung, R., C. Tang, S. Haddad, J. Mcgrenere and P. Graf, 2012. How older adults learn to use mobile devices: Survey and field investigations. ACM Trans. Access. Comput. DOI: $10.1145 / 2399193.2399195$ 
Lindberg, T. and R. Näsänen, 2003 The effect of icon spacing and size on the speed of icon processing in the human visual system. Displays, 24: 111-120. DOI: $10.1016 / \mathrm{S} 0141-9382(03) 00035-0$

Mäkelä, S., 2015. Value of mobile phones for Tanzanian university students. Int. J. Inf. Comm. Technol. Hum. Dev., 7: 57-70.

DOI: 10.4018/IJICTHD.2015040104

Marcus, A., 2013. Design, user experience and usability: Web, mobile and product design. Proceedings of the 2nd International Conference of Design, User Experience and Usability, Jul. 21-26, USA.

McDougall, S.J., O. de Bruijn and M.B. Curry, 2000. Exploring the effects of icon characteristics on user performance: The role of icon concreteness, complexity and distinctiveness. J. Exp. Psychol Appl., 6: 291-306.

DOI: 10.1037/1076-898X.6.4.291

Rogers, Y., 1989. Icon design for the user interface. Int. Revi. Ergon., 3: 129-155.

Rogers, Y. and D.J. Oborne, 1987. Pictorial communication of abstract verbs in relation to human-computer interaction. Bri. J. Psychol., 78: 99-112. DOI: 10.1111/j.2044-8295.1987.tb02229.x

Schröder, S. and M. Ziefle, 2008. Effects of Icon Concreteness and Complexity on Semantic Transparency: Younger vs. Older Users. Proceedings of the 11th International Conference, Computers Helping People with Special Needs, Jul. 9-11, Linz, Austria, pp: 90-97.

DOI 10.1007/978-3-540-70540-6_12
Siek, K.A., Y. Rogers and K.H. Connelly, 2005. Fat finger worries: how older and younger users physically interact with PDAs. Proceedings of the IFIP TC13 International Conference HumanComputer Interaction, Sep. 12-16, Rome, Italy, pp: 267-280. DOI: 10.1007/11555261_24

Stotts, D.B., 1998. The usefulness of icons on the computer interface: effect of graphical abstraction and functional representation on experienced and novice users. Human Factors Ergonom. Soci. Annu. Meet., 42: 453-457.

DOI: $10.1177 / 154193129804200502$

Wiedenbeck, S., 1999. The use of icons and labels in an end user application program: an empirical study of learning and retention. Behaviour Inform. Technol., 18: 68-82. DOI: 10.1080/014492999119129

Wilkinson, C.R., P. Langdon and P.J. Clarkson, 2013. Exploring prior experience and the effects of age on product interaction and learning. Proceedings of the 2nd International Conference of Design, User Experience and Usability, (UEU' 13), Springer pp: 457-466. DOI: 10.1007/978-3-642-39253-5 51

Ziefle, M. and S. Bay, 2005. How older adults meet complexity: aging effects on the usability of different mobile phones. Behaviour Inform. Technol., 24: 375-389.

DOI: $10.1080 / 0144929042000320009$ 